\title{
Effects of Concurrent Training on Oxidative Stress and Insulin Resistance in Obese Individuals
}

\author{
Niara da Silva Medeiros, Fabiana Guichard de Abreu, \\ Alana Schraiber Colato, Leandro Silva de Lemos, Thiago Rozales Ramis, \\ Gilson Pires Dorneles, Cláudia Funchal, and Caroline Dani
}

Centro Universitário Metodista IPA, 90420-060 Porto Alegre, RS, Brazil

Correspondence should be addressed to Caroline Dani; carolinedani@yahoo.com.br

Received 19 November 2014; Revised 7 January 2015; Accepted 15 January 2015

Academic Editor: Vladimir Jakovljevic

Copyright (C) 2015 Niara da Silva Medeiros et al. This is an open access article distributed under the Creative Commons Attribution License, which permits unrestricted use, distribution, and reproduction in any medium, provided the original work is properly cited.

\begin{abstract}
Obesity is associated with insulin resistance (IR) and increased oxidative stress. Thus, the present study aimed to evaluate anthropometric parameters, IR, and oxidative stress in obese individuals subjected to two types of concurrent training at the same intensity but differing in frequency. Accordingly, 25 individuals were divided into two groups: concurrent training 1 (CT1) (5 d/wk) and concurrent training 2 (CT2) ( $3 \mathrm{~d} /$ wk), both with moderate intensity. Anthropometric parameters, IR, and oxidative stress were analyzed before and after 26 sessions of training. Both groups had reduced body weight and body mass index $(P<0.05)$, but only CT1 showed lower body fat percentage and increased basal metabolic rate $(P<0.05)$. Moreover, CT1 had increased HOMA-IR and decreased protein damage (carbonyl level), and CT2 had decreased HOMA-IR and increased lipid peroxidation (TBARS level) $(P<0.05)$. On the other hand, both training protocols reduced the GPx activity. It can be concluded that both types of concurrent training could be an alternative for lowering body weight and BMI. Also, it was observed that concurrent training, depending on the frequency, can contribute to reducing body fat, oxidative damage (protein oxidation), and IR but can induce oxidative damage to lipids. More studies are needed to elucidate the mechanisms involved.
\end{abstract}

\section{Introduction}

Sedentary lifestyle contributes to an increase in the incidence of obesity in most countries [1]. Obesity is defined as a chronic and multifactorial disease, which is associated with high mortality, especially in industrialized areas [1]. This disease is associated with many comorbidities, such as cardiovascular complications, hypertension, atherosclerosis, chronic inflammation, dyslipidemia, insulin resistance (IR), diabetes mellitus (DM), and other metabolic disorders $[1,2]$.

Furthermore, studies show that obesity can cause increased reactive species production and a depletion of antioxidant defenses, leading to oxidative stress. This condition induces oxidative damage to proteins, lipids, and DNA $[3,4]$. Oxidative stress can be reduced by physical exercise with adequate frequency and intensity, which can provide adaptive changes to the regulation of antioxidant defenses, resulting in less oxidative cell damage [5-7].
The benefits of regular physical exercise are well documented [5]. However, there are few studies that approach the benefits of concurrent training (aerobic plus strength) in the obese population. Data demonstrate that this training can help to lose weight and body fat and to gain lean body mass $[7,8]$. However, it is still unclear how concurrent training modulates parameters of IR and oxidative stress in obese individuals. Thus, this study aimed to evaluate anthropometric parameters, IR, and oxidative stress in obese individuals subjected to concurrent training of moderate intensity but differing in frequency.

\section{Materials and Methods}

2.1. Experimental Approach to the Problem. Concurrent training consisted in aerobic exercise combined with strength exercises. The training was divided into two groups: concurrent training 1 (CT1) and concurrent training 2 (CT2). 
TABLE 1: Schedule of concurrent training.

\begin{tabular}{|c|c|c|c|c|c|c|}
\hline & \multicolumn{6}{|c|}{ Sessions } \\
\hline & $1-3^{*}$ & $4-9$ & $10-15$ & $16-21$ & $22-24$ & $25-26$ \\
\hline \multicolumn{7}{|l|}{ Strength exercises } \\
\hline Number of series & & 3 & 3 & 3 & 3 & 3 \\
\hline Number of reps & & 15 & 12 & 10 & 08 & 15 \\
\hline Intensity (\% 1RM) & & $50 \%$ & $60 \%$ & $70 \%$ & $75 \%$ & $50 \%$ \\
\hline Volume per session & & 45 & 36 & 30 & 24 & 45 \\
\hline Recovery time & & $60^{\prime \prime}$ & $90^{\prime \prime}$ & $90^{\prime \prime}$ & $90^{\prime \prime}$ & $60^{\prime \prime}$ \\
\hline \multicolumn{7}{|l|}{ Aerobic exercises } \\
\hline Volume/time & & $30^{\prime}$ & $30^{\prime}$ & $30^{\prime}$ & $30^{\prime}$ & $30^{\prime}$ \\
\hline Intensity $\% \mathrm{VO}_{2 \text { peak }}$ & & $50 \%$ & $60 \%$ & $70 \%$ & $75 \%$ & $50 \%$ \\
\hline Stage Borg scale & & 12 & 13 & 14 & 15 & 12 \\
\hline
\end{tabular}

${ }^{*}$ First 3 sessions to adapt to the exercises.

In both training protocols, the participants were subjected to 26 sessions of 70 minutes each, with the same progressive intensity of $50-75 \% \mathrm{VO}_{2 \text { peak }}$ monitored by the Borg scale (Table 1). Each training session was divided into five minutes of initial warm-up, 30 minutes of walking, 30 minutes of strength exercises, and five minutes of stretching. CT1 was performed five days per week and CT2 three days per week. These different training frequencies were used to check for changes of oxidative profile and insulin resistance in obese sedentary individuals.

2.2. Subjects. The study was conducted in the Centro Universitário Metodista IPA, in southern Brazil. The study included individuals $>18$ years of age, sedentary (no more than 150 minutes of exercise per week) [9], with body mass index (BMI) of $30-40 \mathrm{~kg} / \mathrm{m}^{2}$.

Thus, this study consisted of 25 individuals, 18 women and 7 men. The CT1 group was composed of 8 women and 4 men with age of $45.33 \pm 10.46$ years and height of $161.58 \pm 6.19 \mathrm{~cm}$. The CT2 group was composed of 10 women and 3 men, with age of $49.15 \pm 9.47$ years and height of $163.46 \pm 10.43 \mathrm{~cm}$. The two groups did not differ statistically in baseline values of age, height, and BMI ( $P>0.05)$, indicating homogeneous groups.

The exclusion criteria were subjects who reported a history of autoimmune diseases, cancer, smoking, and diseases that make it impossible to engage in physical exercise and/or had contraindications. All participants read and signed an informed consent form and also had a doctor's permission to perform physical exercise. This study was approved by the Research Ethics Committee of the Centro Universitário Metodista IPA under protocol 37/12.

2.3. Procedures. Before the beginning of the training period, all individuals had a cardiorespiratory test, which consisted of a progressive test until exhaustion on a treadmill (ATL Inbramed Millennium, Porto Alegre, Brazil) with an ergospirometer (V02000, Medgraphics, St. Paul, Minnesota, USA). The test was conducted according to the modified Bruce protocol [9]. The Borg scale was used to monitor the intensity of the test, so that the intensity could be reproduced during physical training. To determine the intensity of exercise and follow a progressive linear intensity, the highest mean oxygen uptake $\left(\mathrm{VO}_{2}\right)$ during 30 seconds was expressed as the peak oxygen uptake $\left(\mathrm{VO}_{2 \text { peak }}\right)$ because a $\mathrm{VO}_{2}$ plateau was invariably not observed during the test, although other criteria for $\mathrm{VO}_{2 \max }$ given in the literature (i.e., RER $>1.1$ and maximum HR within 10 beats of the age-appropriate reference value) were fulfilled [10].

To monitor the intensity of strength exercise, maximal dynamic strength was assessed by the one repetition maximum test (1RM) (following a progressive intensity of 5075\%) [11]. The 1RM test was performed for lower body by squat exercise and for upper body by supine exercise with free weights before starting the training period.

Strength training was based on the method of alternating segments with the following exercises: bench press with dumbbells plan, squats, shoulder abduction, plantar flexion, horizontal pulley, and abdominal crunch. During training, we applied progressive intensity (50-75\% 1RM) as well as decreased volume of repetitions in all exercises, except for abdominal and plantar flexion. The abdominal and plantar flexion exercises were performed with no load and fixed in 15 repetitions.

Anthropometric measurements were assessed before and after the training period, including body weight (kg) (Welmy semianalytical balance), height $(\mathrm{cm})$ (Welmy stadiometer), BMI (defined as the weight (kg) divided by height $(\mathrm{m})$, squared $\mathrm{kg} / \mathrm{m}^{2}$ ), abdomen, waist, and hip circumferences $(\mathrm{cm})$, free fat mass $(\mathrm{kg})$, body fat percentage (\%), and basal metabolic rate (kcal) by the bioelectrical impedance method (Byodinamics BIA 310e).

Moreover, before and after the training period, samples of venous blood were collected $(10 \mathrm{~mL})$ without anticoagulant, after an overnight fast of 8 hours. Serum was separated by centrifugation at $1000 \mathrm{~g}$ for 10 minutes, aliquoted, and frozen at $-20^{\circ} \mathrm{C}$ for further analysis.

We analyzed fasting glucose (automated method, COBAS C111, mg/dL), fasting insulin (DRG Insulin ELISA Kit, $\mu \mathrm{IU} / \mathrm{mL}$ ), and HOMA-IR (homeostasis model of IR) calculated by the formula (fasting insulin $(\mu \mathrm{IU} / \mathrm{mL}) \times$ fasting glucose $(\mathrm{mmol} / \mathrm{L}) / 22.5)$.

To evaluate the oxidative profile of the participants, lipid peroxidation was determined by the thiobarbituric acid reactive substances (TBARS) method described by Wills [12]. Protein carbonyl levels were measured to determine protein oxidation as described by Reznick and Packer [13]. Total sulfhydryl groups were assayed by the technique described by Aksenov and Markesbery [14]. We also determined the activities of the antioxidant enzymes catalase (CAT) [15], superoxide dismutase (SOD) [16], and glutathione peroxidase (GPx) [17].

2.4. Statistical Analysis. All variables were tested for normality of distribution by the Shapiro-Wilk test. For those that showed normality, we used the paired $t$-test for comparison of before and after training (mean \pm standard error). All analyses were performed by the Statistical Package for Social 
TABLE 2: Anthropometric parameters of obese individuals before and after concurrent training with different frequencies.

\begin{tabular}{|c|c|c|c|c|c|c|}
\hline \multirow{2}{*}{$\begin{array}{l}\text { Anthropometric } \\
\text { parameters }\end{array}$} & \multicolumn{3}{|c|}{ CT1 } & \multicolumn{3}{|c|}{ CT2 } \\
\hline & Before & After & $P$ & Before & After & $P$ \\
\hline Body weight (kg) & $96.06 \pm 4.32$ & $93.34 \pm 4.26$ & $<0.001$ & $89.98 \pm 4.64$ & $87.20 \pm 4.30$ & 0.013 \\
\hline BMI $\left(\mathrm{kg} / \mathrm{m}^{2}\right)$ & $36.73 \pm 1.60$ & $35.68 \pm 1.55$ & $<0.001$ & $33.53 \pm 1.03$ & $32.55 \pm 1.00$ & 0.011 \\
\hline FFM (kg) & $59.59 \pm 2.90$ & $61.74 \pm 3.25$ & 0.021 & $56.41 \pm 3.68$ & $57.27 \pm 4.27$ & 0.541 \\
\hline $\mathrm{BF}(\%)$ & $37.35 \pm 1.57$ & $33.78 \pm 1.91$ & $<0.001$ & $38.11 \pm 0.98$ & $36.68 \pm 1.39$ & 0.062 \\
\hline $\mathrm{WC}(\mathrm{cm})$ & $102.94 \pm 2.94$ & $101.91 \pm 2.87$ & 0.177 & $97.77 \pm 2.55$ & $95.11 \pm 2.68$ & 0.077 \\
\hline $\mathrm{AC}(\mathrm{cm})$ & $113.27 \pm 3.15$ & $111.14 \pm 2.79$ & 0.074 & $105.33 \pm 1.69$ & $101.26 \pm 1.25$ & 0.026 \\
\hline $\mathrm{HC}(\mathrm{cm})$ & $117.55 \pm 2.27$ & $113.23 \pm 1.99$ & $<0.001$ & $113.79 \pm 2.01$ & $111.16 \pm 1.80$ & 0.007 \\
\hline BMR (kcal) & $1825.82 \pm 87.00$ & $1872.36 \pm 99.31$ & 0.033 & $1715.77 \pm 111.94$ & $1735.54 \pm 129.26$ & 0.651 \\
\hline
\end{tabular}

Variables presented as mean \pm standard error. CT1: concurrent training 5 times per week; CT2: concurrent training 3 times per week. BMI: body mass index; FFM: free fat mass; BF\%: body fat percentage, WC: waist circumference; AC: abdominal circumference; HC: hip circumference; BMR: basal metabolic rate. $P<0.05$ (significantly different). Paired $t$-test was utilized to compare before and after training in the same group.

TABLE 3: Comparison of markers of insulin resistance and oxidative stress in obese individuals before and after concurrent training.

\begin{tabular}{|c|c|c|c|c|c|c|}
\hline & \multicolumn{3}{|c|}{ CT1 } & \multicolumn{3}{|c|}{ CT2 } \\
\hline & Before & After & $P$ & Before & After & $P$ \\
\hline \multicolumn{7}{|l|}{ Insulin resistance markers } \\
\hline Fasting glucose (mg/dL) & $96.50 \pm 3.85$ & $85.66 \pm 4.13$ & 0.096 & $100.96 \pm 3.76$ & $85.69 \pm 4.13$ & 0.001 \\
\hline Fasting insulin $(\mathrm{uUI} / \mathrm{mL})$ & $10.54 \pm 0.84$ & $14.29 \pm 0.77$ & 0.002 & $9.55 \pm 0.77$ & $8.54 \pm 0.64$ & 0.293 \\
\hline HOMA-IR & $2.56 \pm 0.26$ & $3.77 \pm 0.35$ & 0.005 & $2.34 \pm 0.56$ & $1.79 \pm 0.52$ & 0.009 \\
\hline \multicolumn{7}{|l|}{ Oxidative stress markers } \\
\hline TBARS (nmol/mL) & $3.69 \pm 0.31$ & $3.69 \pm 0.11$ & 0.987 & $4.70 \pm 0.17$ & $6.35 \pm 0.049$ & 0.015 \\
\hline Carbonyl (nmol DNPH/mg) & $116.36 \pm 18.36$ & $34.45 \pm 7.46$ & 0.012 & $24.96 \pm 9.67$ & $24.39 \pm 8.57$ & 0.965 \\
\hline Sulfhydryl ( $\mu \mathrm{M} / \mathrm{mg})$ & $11.83 \pm 11.03$ & $9.03 \pm 1.23$ & 0.145 & $5.20 \pm 1.04$ & $4.09 \pm 0.41$ & 0.169 \\
\hline CAT (UCAT/mg) & $2.79 \pm 0.66$ & $4.73 \pm 1.24$ & 0.281 & $3.26 \pm 0.63$ & $1.83 \pm 0.28$ & 0.074 \\
\hline $\mathrm{SOD}(\mathrm{USOD} / \mathrm{mg}$ & $1.85 \pm 0.50$ & $2.40 \pm 0.86$ & 0.667 & $1.29 \pm 0.46$ & $3.07 \pm 1.38$ & 0.235 \\
\hline GPx (UGPx/mg) & $3.86 \pm 0.40$ & $3.37 \pm 0.37$ & $<0.001$ & $4.57 \pm 0.45$ & $4.22 \pm 0.42$ & 0.002 \\
\hline
\end{tabular}

Variables presented as mean \pm standard error. CT1: concurrent training 5 times per week; CT2: concurrent training 3 times per week. HOMA-IR: homeostasis model of insulin resistance; TBARS: thiobarbituric acid reactive substances; CAT: catalase activity; SOD: superoxide dismutase activity; GPx: glutathione peroxidase activity.

$P<0.05$ (significantly different). Paired $t$-test was utilized to compare before and after training in the same group.

Sciences (SPSS) program version 18.0. All statistical tests were two-tailed and performed using a significance level of $\alpha=$ 0.05 .

\section{Results}

It was observed that both training protocols were able to reduce body weight and BMI (Table 2). But only CT1 showed significantly decreased body fat percentage and increased free fat mass and BMR after the training period. Regarding the circumference measurements, hip circumference was decreased in CT1, and abdomen and hip circumferences were decreased in CT2 (Table 2).

With regard to IR parameters, the CT1 group showed a significant increase in fasting insulin level and HOMA-IR and no change in fasting glucose. However, the CT2 group showed a significant decrease in fasting glucose level and HOMA-IR, with no change in fasting insulin (Table 3).

Finally, for oxidative stress markers, only carbonyls and GPx activity were reduced in the CT1 group, while, in the CT2 group, the TBARS levels increased and GPx activity decreased (Table 3).

\section{Discussion}

Exercise is a strong ally in the treatment of obesity, and when performed with adequate intensity and frequency, it could provide protection against comorbidities of obesity. This includes improvement in the cardiovascular system (reduced blood pressure, increased peripheral blood flow, reduced atherosclerosis progression, and decreased oxygen demand of the myocardium), reduced risk of developing type $2 \mathrm{DM}$ (by reducing IR), anxiety, and depression, and increased metabolic rate [18-20].

It is known that aerobic exercise is beneficial in weight loss, modulation of oxidative stress, and reduction of IR in obese individuals [21,22]. But there are few studies applying the strength exercise associated with aerobic exercise (concurrent training) in this population. This training can help to reduce body weight and fat mass by increasing lean mass and basal metabolic rate [23-26].

These benefits were observed in this study, and both forms of concurrent trainings were able to reduce body weight and BMI. CT1 ( $5 \mathrm{x} /$ week) also reduced the body fat percentage and hip circumference and even increased basal metabolic rate. These findings were also shown in a study by Willis et al. [7] 
who demonstrated a reduction in weight, body fat percentage, and waist circumference in overweight adults who performed concurrent training $3 \mathrm{x} /$ week with an intensity of $65-80 \%$ $\mathrm{VO}_{2 \text { peak }}$.

Another study also showed that overweight individuals who performed concurrent training for 12 weeks, 5 days/week, had a decrease in weight, BMI, body fat percentage, and waist circumference. However, in this same study, two other groups that performed strength training or aerobic training only had a decrease in waist circumference, showing the relevance of the type of training [6].

Our study also demonstrated a significant increase in free fat mass in CT1, which can result in neuromuscular adaptations such as increased muscle mass and maximal strength dynamic capacity and can improve basal metabolic rate in obese individuals. Also, other studies have shown that concurrent training has positive effects in the development of strength and muscle mass in both healthy young individuals [27] and overweight dyslipidemic individuals [28].

Furthermore, according to the IR data presented, the CT1 group showed an increase in fasting insulin level and HOMAIR, although this increase was still within normal range $(2-25 \mu \mathrm{IU} / \mathrm{mL})$. On the other hand, CT2 reduced glucose levels and HOMA-IR but did not alter fasting insulin. In the literature, there are few studies demonstrating the role of concurrent training in obese nondiabetics. But a study of subjects with metabolic syndrome and type 2 DM showed that concurrent training for 20 weeks at $70-80 \% \mathrm{VO}_{2 \text { peak }}$ reduced HOMA-IR as well as weight and waist circumference [29] starting on the third week, thereby corroborating our findings.

Studies have shown a direct relationship between exercise and insulin sensitivity $[29,30]$. However, a short time of physical activity is associated with low insulin sensitivity, and a few days of rest are associated with increased IR [2931]. Thus, regular exercise can improve insulin sensitivity in healthy subjects and obese nondiabetics [19, 32-34]. Notably, the positive effect of exercise on insulin sensitivity has been demonstrated 12-48 hours after the last session of exercise, but preexercise levels return in three or five days [35], highlighting the importance of regular exercise.

IR is associated with obesity, and both are related to oxidative stress. Clinical and animal models studies have shown that oxidative stress is a potential mediator of IR, particularly in skeletal muscle, because there is an inverse relationship between oxidative stress and insulin action [3638]. Prolonged exposure to low levels of oxidative stress reduces insulin sensitivity (reduction in GLUT-4) [39] causing defects in its signaling $[40,41]$.

According to a study in a rat model of insulin-resistant obesity (Zucker), endurance training reduced protein oxidation (carbonyl) levels after training [36]. This research group also reported that this exercise improved insulin action and glucose transport in the soleus, plantar, and cardiac muscles [37]. These results are in line with our findings in concurrent training, that is, reduced damage to proteins (carbonyls). Furthermore, another study with Wistar rats showed a decrease in lipid peroxidation (TBARS), increase in antioxidant enzyme SOD activity, and reduction in triglyceride levels when concurrent training was performed $5 \mathrm{x} /$ week. It was also observed that this improvement in the antioxidant system was similar in the groups that performed exclusively aerobic or strength training [42].

Furthermore, the CT2 group showed lower GPx activity and a trend $(P=0.07)$ towards a reduction in CAT activity but no change in SOD activity and sulfhydryl level, which may have contributed to the increase in TBARS in this group. It is well known that exercise promotes increased oxidative stress, because during exercise the rate of oxygen consumption increases about 20 times, and in exhaustive exercise glutathione activity can be reduced [43]. Perhaps, the intensity and frequency of CT2 were not sufficient to modulate antioxidant defenses and thereby induce the necessary adjustments to reduce damage to lipids.

Many tissues can produce reactive oxygen species during physical exercise. Studies have investigated which organs are primarily responsible for reactive oxygen species production in exercising humans. The lack of in vivo studies on this topic is due to the difficulty of investigating the multifaceted nature of exercise, which involves several organ systems, and these organs are connecting through the increased energy requirement of contracting skeletal muscles [44]. Investigators have assumed that skeletal muscle provides the major source of free radical and reactive oxygen species generation during exercise [44]. Nonetheless, other tissues such as the heart, lungs, or blood may also contribute to the total body generation of reactive oxygen species during exercise [44, 45].

Studies have shown that prolonged or intense contractile activity alters the physiological environment in muscle fibers resulting in conditions that predispose these fibers to higher rates of reactive oxygen species production. Increased oxygen consumption in muscle fibers lowers intracellular oxygen tension during exercise, which can promote increased reactive oxygen species production [46]. Further, a rise in muscle temperature, increased $\mathrm{CO}_{2}$ tension, and decreased cellular $\mathrm{pH}$ are other exercise-related changes that can stimulate reactive oxygen species production in muscle. These contractioninduced changes can stimulate superoxide production at multiple subcellular sites $[46,47]$.

On the basis of this study, concurrent training may be an alternative to lowering weight and BMI, but when training is performed more frequently (5 times a week), these benefits can be enhanced by reducing body fat and protein damage as well. On the other hand, the effects of such training on oxidative stress parameters and IR were contradictory, and thus more studies are needed to elucidate the mechanisms of concurrent training in this population.

\section{Conflict of Interests}

The authors have no conflict of interests to disclose in this study.

\section{Acknowledgments}

The authors are grateful to the study participants for their cooperation and collaborators for their dedication. 
The financial support was provided by Centro Universitário Metodista, do IPA, and Coordenação de Aperfeiçoamento de Pessoal de Nível Superior (CAPES). Dr. A. Leyva helped with English editing of the paper.

\section{References}

[1] S. Jebb, "Obesity: causes and consequences," Women's Health Medicine, vol. 1, no. 1, pp. 38-41, 2004.

[2] F. Amirkhizi, F. Siassi, M. Djalali, and A. R. Foroushani, "Evaluation of oxidative stress and total antioxidant capacity in women with general and abdominal adiposity," Obesity Research and Clinical Practice, vol. 4, no. 3, pp. e209-e216, 2010.

[3] I. Grattagliano, V. O. Palmieri, P. Portincasa, A. Moschetta, and G. Palasciano, "Oxidative stress-induced risk factors associated with the metabolic syndrome: a unifying hypothesis," The Journal of Nutritional Biochemistry, vol. 19, no. 8, pp. 491-504, 2008.

[4] S. Furukawa, T. Fujita, M. Shimabukuro et al., "Increased oxidative stress in obesity and its impact on metabolic syndrome," The Journal of Clinical Investigation, vol. 114, no. 12, pp. 1752-1761, 2004.

[5] R. E. Andersen and J. M. Jakicic, "Interpreting the physical activity guidelines for health and weight management," Journal of Physical Activity and Health, vol. 6, no. 5, pp. 651-656, 2009.

[6] S. S. Ho, S. S. Dhaliwal, A. P. Hills, and S. Pal, "The effect of 12 weeks of aerobic, resistance or combination exercise training on cardiovascular risk factors in the overweight and obese in a randomized trial," BMC Public Health, vol. 12, article 704, 2012.

[7] L. H. Willis, C. A. Slentz, L. A. Bateman et al., "Effects of aerobic and/or resistance training on body mass and fat mass in overweight or obese adults," Journal of Applied Physiology, vol. 113, no. 12, pp. 1831-1837, 2012.

[8] E. Ghahramanloo, A. W. Midgley, and D. J. Bentley, "The effect of concurrent training on blood lipid profile and anthropometrical characteristics of previously untrained men," Journal of Physical Activity and Health, vol. 6, no. 6, pp. 760-766, 2009.

[9] American College of Sports Medicine (ACSM), ACSM's Guidelines for Exercise Testing and Prescription, Lippincott Williams \& Wilkins, Baltimore, Md, USA, 8th edition, 2009.

[10] E. T. Howley, D. R. Bassett Jr., and H. G. Welch, "Criteria for maximal oxygen uptake: review and commentary," Medicine and Science in Sports and Exercise, vol. 27, no. 9, pp. 1292-1301, 1995.

[11] L. A. Brown, C. J. Kerr, P. Whiting, N. Finer, J. McEneny, and T. Ashton, "Oxidant stress in healthy normal-weight, overweight and obese individuals," Obesity, vol. 17, no. 3, pp. 460-466, 2009.

[12] E. D. Wills, "Mechanisms of lipid peroxide formation in animal tissues.," Biochemical Journal, vol. 99, no. 3, pp. 667-676, 1966.

[13] A. Z. Reznick and L. Packer, "Oxidative damage to proteins: spectrophotometric method for carbonyl assay," Methods in Enzymology, vol. 233, pp. 357-363, 1994.

[14] M. Y. Aksenov and W. R. Markesbery, "Changes in thiol content and expression of glutathione redox system genes in the hippocampus and cerebellum in Alzheimer's disease," Neuroscience Letters, vol. 302, no. 2-3, pp. 141-145, 2001.

[15] H. Aebi, "[13] Catalase in vitro," Methods in Enzymology, vol. 105, pp. 121-126, 1984.

[16] H. P. Misra and I. Fridovich, "The role of superoxide anion in the autoxidation of epinephrine and a simple assay for superoxide dismutase," The Journal of Biological Chemistry, vol. 247, no. 10, pp. 3170-3175, 1972.

[17] A. Wendel, "Glutathione peroxidase," Methods in Enzymology, vol. 77, pp. 325-333, 1981.

[18] T. S. Church, C. P. Earnest, J. S. Skinner, and S. N. Blair, "Effects of different doses of physical activity on cardiorespiratory fitness among sedentary, overweight or obese postmenopausal women with elevated blood pressure," The Journal of the American Medical Association, vol. 297, no. 19, pp. 2081-2091, 2007.

[19] D. M. Okay, P. V. Jackson, M. Marcinkiewicz, and M. N. Papino, "Exercise and obesity," Primary Care-Clinics in Office Practice, vol. 36, no. 2, pp. 379-393, 2009.

[20] D. E. R. Warburton, C. W. Nicol, and S. S. D. Bredin, "Health benefits of physical activity: the evidence," Canadian Medical Association Journal, vol. 174, no. 6, pp. 801-809, 2006.

[21] M. F. F. Mediano and R. Sichieri, "Insulin resistance influences weight loss in non-obese women who followed a homebased exercise program and slight caloric restriction," Diabetes Research and Clinical Practice, vol. 92, no. 3, pp. 361-367, 2011.

[22] G. Murdolo, M. Piroddi, F. Luchetti et al., "Oxidative stress and lipid peroxidation by-products at the crossroad between adipose organ dysregulation and obesity-linked insulin resistance," Biochimie, vol. 95, no. 3, pp. 585-594, 2013.

[23] A. Chatzinikolaou, I. Fatouros, A. Petridou et al., "Adipose tissue lipolysis is upregulated in lean and obese men during acute resistance exercise," Diabetes Care, vol. 31, no. 7, pp. 13971399, 2008.

[24] M. J. Ormsbee, D. C. Myung, J. K. Medlin et al., "Regulation of fat metabolism during resistance exercise in sedentary lean and obese men," Journal of Applied Physiology, vol. 106, no. 5, pp. 1529-1537, 2009.

[25] H. T. Rabelo, L. A. Bezerra, D. F. Terra et al., "Effects of 24 weeks of progressive resistance training on knee extensors peak torque and fat-free mass in older women," The Journal of Strength and Conditioning Research, vol. 25, no. 8, pp. 2298-2303, 2011.

[26] R. A. Washburn, E. P. Kirk, B. K. Smith et al., "One set resistance training: effect on body composition in overweight young adults," Journal of Sports Medicine and Physical Fitness, vol. 52, no. 3, pp. 273-279, 2012.

[27] S. P. Glowacki, S. E. Martin, A. Maurer, W. Baek, J. S. Green, and S. F. Crouse, "Effects of resistance, endurance, and concurrent exercise on training outcomes in men," Medicine and Science in Sports and Exercise, vol. 36, no. 12, pp. 2119-2127, 2004.

[28] M. I. Schaun, T. Dipp, J. S. Rossato et al., "The effects of periodized concurrent and aerobic training on oxidative stress parameters, endothelial function and immune response in sedentary male individuals of middle age," Cell Biochemistry and Function, vol. 29, no. 7, pp. 534-542, 2011.

[29] S. Balducci, S. Zanuso, A. Nicolucci et al., "Anti-inflammatory effect of exercise training in subjects with type 2 diabetes and the metabolic syndrome is dependent on exercise modalities and independent of weight loss," Nutrition, Metabolism and Cardiovascular Diseases, vol. 20, no. 8, pp. 608-617, 2010.

[30] K. L. Rennie, N. McCarthy, S. Yazdgerdi, M. Marmot, and E. Brunner, "Association of the metabolic syndrome with both vigorous and moderate physical activity," International Journal of Epidemiology, vol. 32, no. 4, pp. 600-606, 2003.

[31] T. A. Lakka, D. E. Laaksonen, H.-M. Lakka et al., "Sedentary lifestyle, poor cardiorespiratory fitness, and the metabolic syndrome," Medicine and Science in Sports \& Exercise, vol. 35, no. 8, pp. 1279-1286, 2003. 
[32] S. E. Kahn, V. G. Larson, J. C. Beard et al., "Effect of exercise on insulin action, glucose tolerance, and insulin secretion in aging," The American Journal of Physiology -Endocrinology and Metabolism, vol. 258, no. 6, pp. E937-E943, 1990.

[33] J. P. Kirwan, W. M. Kohrt, D. M. Wojta, R. E. Bourey, and J. O. Holloszy, "Endurance exercise training reduces glucosestimulated insulin levels in 60- to 70-year-old men and women," The Journals of Gerontology, vol. 48, no. 3, pp. M84-M90, 1993.

[34] J. P. Miller, R. E. Pratley, A. P. Goldberg et al., "Strength training increases insulin action in healthy 50- to 65-yr-old men," Journal of Applied Physiology, vol. 77, no. 3, pp. 1122-1127, 1994.

[35] J. Eriksson, S. Taimela, and V. A. Koivisto, "Exercise and the metabolic syndrome," Diabetologia, vol. 40, no. 2, pp. 125-135, 1997.

[36] V. Saengsirisuwan, T. R. Kinnick, M. B. Schmit, and E. J. Henriksen, "Interactions of exercise training and lipoic acid on skeletal muscle glucose transport in obese Zucker rats," Journal of Applied Physiology, vol. 91, no. 1, pp. 145-153, 2001.

[37] M. K. Teachey, Z. C. Taylor, T. Maier et al., "Interactions of conjugated linoleic acid and lipoic acid on insulin action in the obese Zucker rat," Metabolism: Clinical and Experimental, vol. 52, no. 9, pp. 1167-1174, 2003.

[38] H. Urakawa, A. Katsuki, Y. Sumida et al., "Oxidative stress is associated with adiposity and insulin resistance in men," The Journal of Clinical Endocrinology and Metabolism, vol. 88, no. 10, pp. 4673-4676, 2003.

[39] A. Rudich, N. Kozlovsky, R. Potashnik, and N. Bashan, "Oxidant stress reduces insulin responsiveness in 3T3-L1 adipocytes," The American Journal of Physiology-Endocrinology and Metabolism, vol. 272, no. 5, pp. E935-E940, 1997.

[40] B. A. Maddux, W. See, J. C. Lawrence Jr., A. L. Goldfine, I. D. Goldfine, and J. L. Evans, "Protection against oxidative stressinduced insulin resistance in rat L6 muscle cells by micromolar concentrations of $\alpha$-lipoic acid," Diabetes, vol. 50, no. 2, pp. 404410, 2001.

[41] A. Rudich, A. Tlrosh, R. Potashnik, R. Hemi, H. Kanety, and N. Bashan, "Prolonged oxidative stress impairs insulin-induced GLUT4 translocation in 3T3-L1 adipocytes," Diabetes, vol. 47, no. 10, pp. 1562-1569, 1998.

[42] J. D. Botezelli, L. T. Cambri, A. C. Ghezzi et al., "Different exercise protocols improve metabolic syndrome markers, tissue triglycerides content and antioxidant status in rats," Diabetology and Metabolic Syndrome, vol. 3, no. 1, article 35, 2011.

[43] L. L. Ji and R. Fu, "Responses of glutathione system and antioxidant enzymes to exhaustive exercise and hydroperoxide," The Journal of Applied Physiology, vol. 72, no. 2, pp. 549-554, 1992.

[44] S. K. Powers and M. J. Jackson, "Exercise-induced oxidative stress: cellular mechanisms and impact on muscle force production," Physiological Reviews, vol. 88, no. 4, pp. 1243-1276, 2008.

[45] M. G. Nikolaidis and A. Z. Jamurtas, "Blood as a reactive species generator and redox status regulator during exercise," Archives of Biochemistry and Biophysics, vol. 490, no. 2, pp. 77-84, 2009.

[46] L. F. Ferreira and M. B. Reid, "Muscle-derived ROS and thiol regulation in muscle fatigue," Journal of Applied Physiology, vol. 104, no. 3, pp. 853-860, 2008.

[47] S. K. Powers, W. B. Nelson, and M. B. Hudson, "Exerciseinduced oxidative stress in humans: cause and consequences," Free Radical Biology \& Medicine, vol. 51, no. 5, pp. 942-950, 2011. 


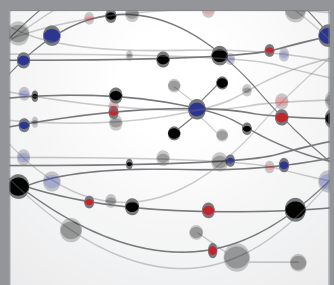

The Scientific World Journal
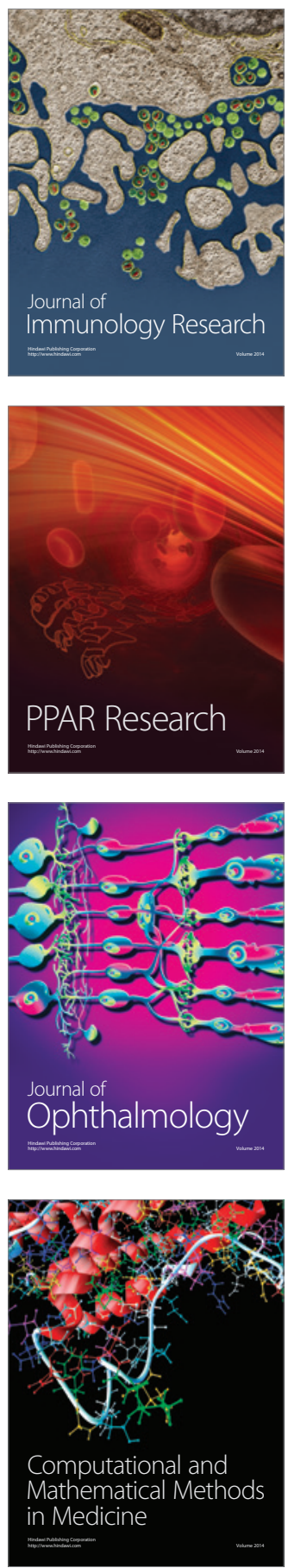

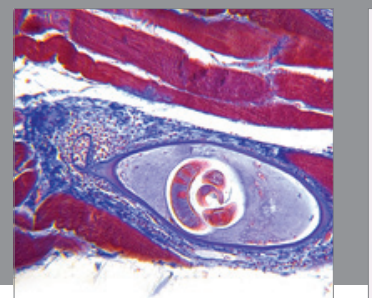

Gastroenterology

Research and Practice
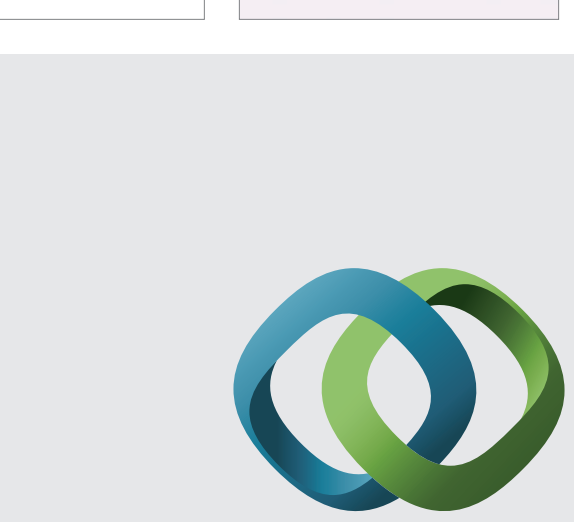

\section{Hindawi}

Submit your manuscripts at

http://www.hindawi.com
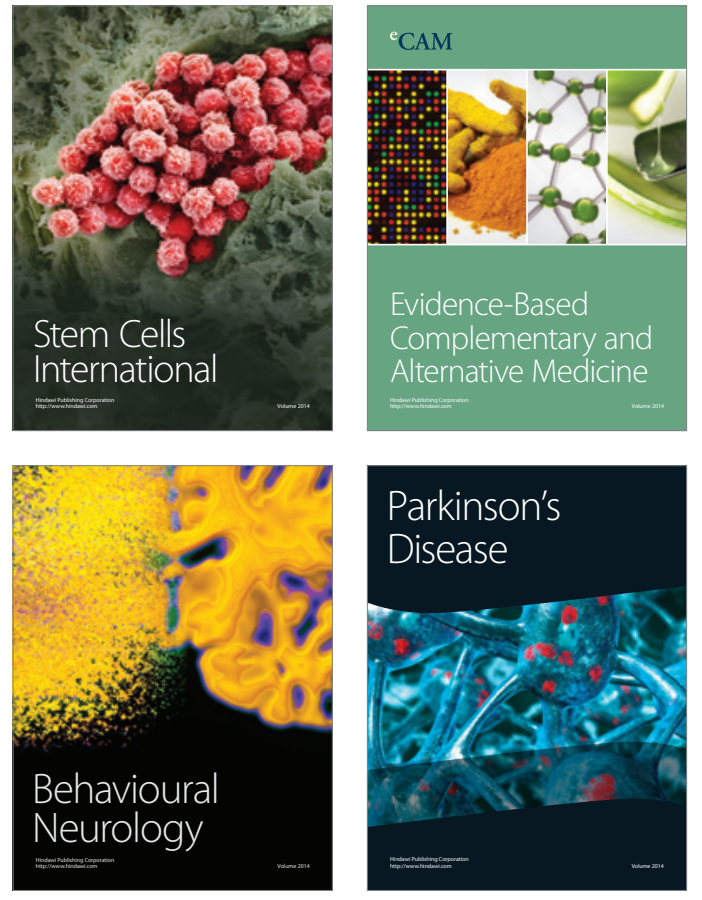
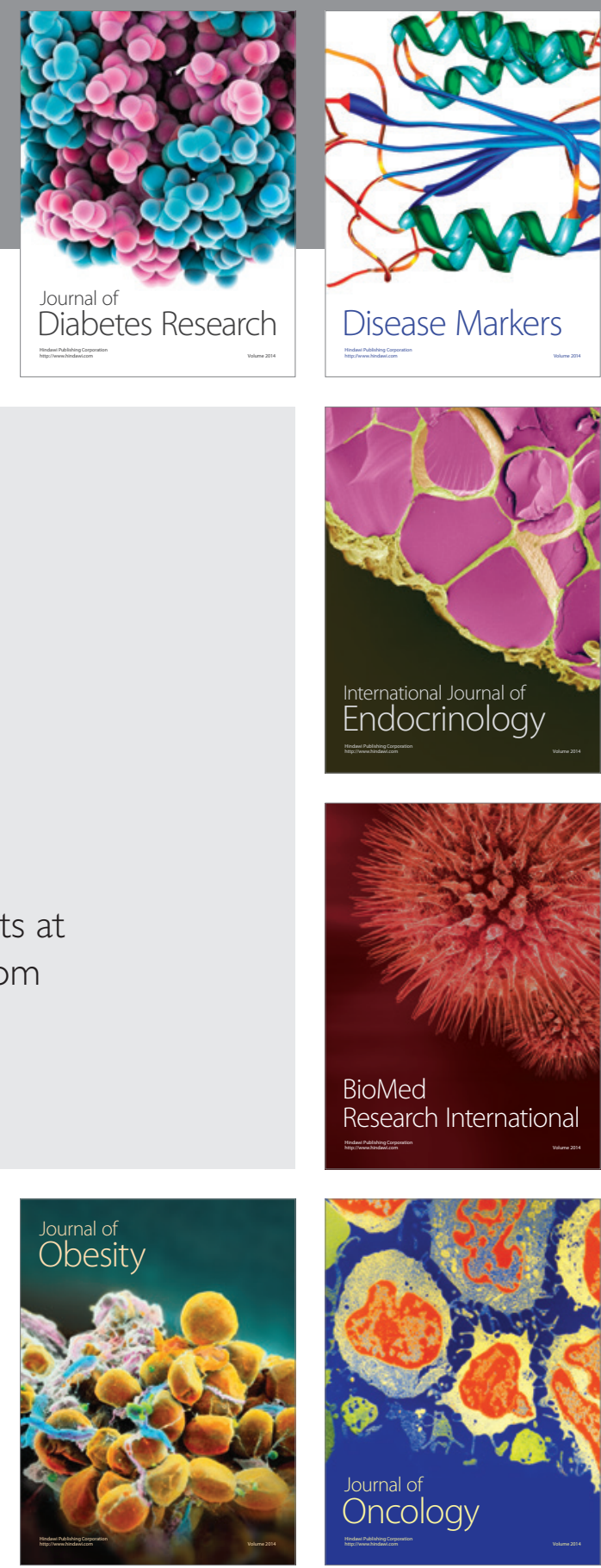

Disease Markers
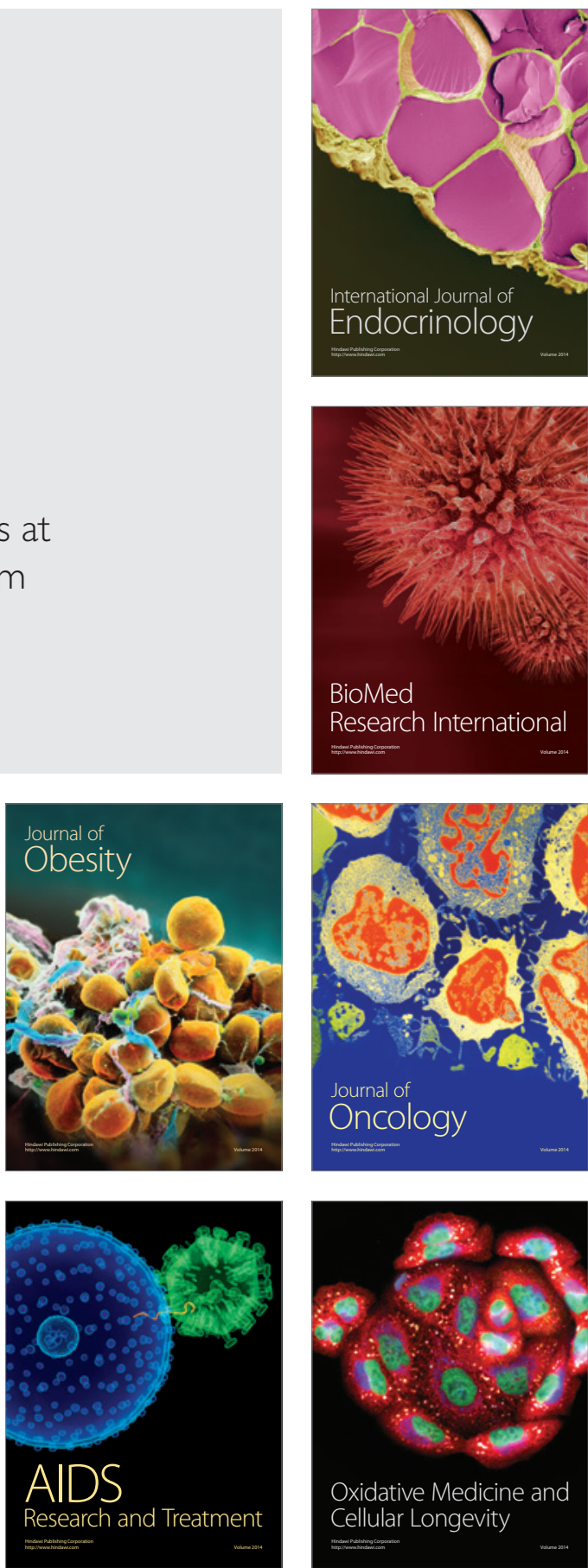